\title{
Emotional responses to different flavoured popcorns on watching different types of movies
}

\author{
*Khajarern, K. \\ Faculty of Interdisciplinary studies, Khon Kaen University, Nong Khai Campus, Nong Khai 43000 Thailand
}

\author{
Article history: \\ Received: 1 June 2021 \\ Received in revised form: 1 \\ July 2021 \\ Accepted: 3 October 2021 \\ Available Online: 28 \\ November 2021
}

Keywords:

Emotional response,

Flavoured popcorns,

Movies,

Emotional ratio profile

DOI:

https://doi.org/10.26656/fr.2017.5(6).377

\begin{abstract}
The emotional responses to five flavoured popcorns (butter, cheese, caramel, barbecue and Thai spicy) compared to 'not eat anything' on watching each genre of five digital video disk (DVD) movies (action, comedy, fantasy, romance and horror movies) were measured by 100 voluntary audiences. They were recruited to participate in the sensory laboratory set as a theatre. After five minutes of watching each movie, they had to taste each of the flavoured popcorns for $25 \mathrm{~g}$ serving with $250 \mathrm{~mL}$ of drinking water than their 39 emotional and 2 sensory responses were recorded. Analysis of variance (ANOVA) and emotional ratio profiling of all flavoured popcorns on watching each movie were analysed. The results revealed that the mean overall liking score for each flavoured popcorn was not different, but the mean emotional scores that responded to each flavoured popcorn on watching each movie were different significantly $(p<0.01)$. During watching the action movie, butter and cheese flavoured popcorns seemed to significantly evoke most emotions on 'daring' and 'pleasant' $(p<0.01)$. While watching the horror movie, caramel flavoured popcorn undoubtedly evoked the most emotional responses among other flavoured popcorns. These emotional responses to each of flavoured popcorns on each of variety DVD movies might be used for movie theatre strategic promotion and advertisement to add value and distinction for the flavoured popcorn products in all cases.
\end{abstract}

\section{Introduction}

The beneficial effects of mood and emotions are believed to influence consumers' behaviour as a food choice (Barthomeuf et al., 2009). Identifying the effects of food on mood and emotion may be of considerable commercial value in product development and marketing (Aschemann-Witzel et al., 2012). Rogers (1996) proposed the interrelationships between diet and behaviour that dietary influences on mood and emotion, and appetite are mediated by physiological mechanisms and may influence food preference, food choice and food intake. This relationship is also needed to the food industry which now produces many products with the same liking ratings and is turning to mood and emotion as another way to distinguish the products. Thus, many food researchers are interested in the scientific studies of food or constituent effects on mood and emotion (Cardello et al., 2012; Khajarern et al., 2010; Khajarern, 2013). These beneficial effects will be used for food marketing strategies such as promotion and advertisement which can potentially increase product sales.
The senses play an important role in consumer perceptions and can have a powerful influence over purchasing decision making. Food marketers have long tried to incorporate the senses into brand communications, although it was in a limited and partial way. Today, sensory marketing is recognized as an essential tool for enhancing the relationship between brand and consumer by stimulating all the senses and generating emotions (Manzano et al., 2019). Hence, movie watching was also influenced by mood (Payne, Shaw and Caldwell, 1997) and emotion (Wiley, 2003). If some food goes well with the movies it should lead to a positive mood and emotion.

Popcorn is a whole-grain food, an important snack food made of maize (Ceylan and Karababa, 2004) and represents one of the most popular snack foods in Thailand (Bangsuntear, 2020) and in a large part of the World (Soylu and Tekkanat, 2007). It is usually sold in general theatres (Wettlaufer and Lemke, 2013) much more than potato chips (Nguyen et al., 2012). Popcorn is a special kind of flint maize selected by Indians in early western civilizations (Arendt and Zannini, 2013). It 
became Western-style convenience food in Asia (Curtis et al., 2007). While the movie theatre business is growing, popcorn is becoming a movie theatre's favourite snack for Americans. It generates a high profit along with the movie theatre business for a long time. Popcorn concession in movie theatre generates revenues of 10 million US dollars and theatre business has a market value of up to 125 million US dollars in Thailand (Bangsuntear, 2020). The snack food business had a market value of 843.7 million US dollars in which popcorn accounts for 71.8 million US dollars (Wunsch, 2020). Now a day, instant popcorn that can be cooked through a microwave is available for television audiences at home (Lago et al., 2013; Bangsuntear, 2020). This indicates that popcorn is an interesting product case for this food emotional research because it is very popular and related to entertainment.

Specifically flavoured popcorns were selected to consume based on each type of movie in the theatres (Singh and Singh, 1999). It is interesting to measure emotional response to the consumption of each popcorn flavour during each genre of movie watched. The question is which flavoured popcorn matches which movie and be able to satisfy audiences as entertainment. Thus, this research is conducted to study the relationship between emotional effects on diets between popcorns and audience preferences. In addition, the emotional responses (obtained from a 39 item list of food-related emotions) (King and Meiselman, 2010) were also tested on each of flavoured popcorns on a variety of digital video disks (DVD) movies.

The movie-watching had significant effects on mood induction (Jampour et al., 2019). But emotional responses to food through flavoured popcorns consumption in the different genres of movies has never been investigated before. This research should benefit the food manufacturers on the mood and emotion of consumers against food in the future.

\section{Materials and methods}

\subsection{Subjects}

One hundred audience volunteers [Resurreccion (1998) recommended consumer panel size for various consumer sensory testing laboratories], of which 50 males and 50 females (aged between 18 and 35 years old) were recruited by using purposive sampling at Khon Kaen University, Nongkhai Campus. They were lecturers, technicians, supporting staff and students who had ever bought popcorn to consume in the theatres. These volunteers had never been trained in a tasting before. The individual had good health and be willing to participate in this study.

\subsection{Sample preparation}

A $100 \mathrm{~g}$ of dry maize kernels (McGarrett American popcorn brand, USA) were popped using a popcorn making machine (F801 Fengshe brand of Guangzhou Fengshen Food Machine Co., Ltd., China) for 30 mins together with $40 \mathrm{~g}$ of butter and $2 \mathrm{~g}$ of fine iodized table salt. Then they were mixed with $50 \mathrm{~g}$ of each flavoured powder (butter, cheese, caramel, Thai spicy (Tom yum) and barbecue) in the stainless steel mixing bowl and were kept warm in the hot displayed cabinet which was controlled temperature at $60 \pm 2^{\circ} \mathrm{C}$ (maintained automatically). They were each packed and closed in a 25 g plastic container before serving.

\subsection{Emotional scale questionnaire}

A set of 41 emotional items consisted of 39 emotional items (King and Meiselman, 2010) and two sensory items, thirst and overall liking (Khajarern, 2013). They were used as the emotional scale items for questionnaire construction (Cronbach's alpha $=0.87$ ). Each volunteer was asked to rate the perception level of their feeling along with these emotions and sensory attributes on "how that flavoured popcorn makes you feel when you eat it". Each emotion and sensory items were rated on a five-point scale $(1=$ not at all, $2=$ slightly, $3=$ moderately, $4=$ very and $5=$ extremely) (Symmank et al., 2018). The questionnaire was placed on a rigid plastic board that could be placed on their laps.

\subsection{Consumer study}

A session of 30-minute movie watching was set to generate the volunteers' emotion (Vorderer, Knobloch and Schramm, 2001) which was required in this study. The main objective of the watching was to induce a state of emotion (Rogers, Green and Edwards, 1994). Twentyfive volunteers (There were 4 sessions per day; 25 volunteers per session) were recruited to the sensory room to watch each of 5 DVD movie genres (5 movie types; action, comedy, fantasy, romance and horror). In each movie, a series of five different short episodes in the same story was used to control the similar experiments (one treatment per one episode). Before testing, they had been informed about the meanings of each emotional item in the questionnaire. The previous researches were performed in English so they were translated into Thai by three linguistic experts. After watching a movie for five minutes, $25 \mathrm{~g}$ of each flavoured popcorn and $250 \mathrm{~mL}$ of drinking water were served (There were 6 treatments per experiment; butter, cheese, caramel, Tom Yum and barbecue flavoured popcorns and 'not eat anything). Hence, each volunteer had to test once (one treatment) a day (6 days per experiment, for 30 days). After tasting, they had to 
indicate the level of their emotion in the questionnaires.

All volunteers were asked not to consume food and drink before and during the test. The Randomized Complete Block Design (RCBD) was assigned for arranging the test for 6 treatments (6 days) for each session and each situation (Meilgaard et al., 2006). The sensory room of Khon Kaen University, Nongkhai Campus was applied as a theatre simulation. It consisted of a projector, white screen (120 inches), DVD movies and DVD player. Twenty-five seats with room temperature at $25^{\circ} \mathrm{C}$ were prepared (Khajarern, 2013).

\subsection{Statistical analysis}

Cronbach's alpha of the questionnaire was analysed for internal consistency reliability and Analysis of Variance (ANOVA) $(p \leq 0.05)$ was also analysed using SPSS/PC for Windows version 26.0. The level of statistical significance was set between $0.01(p<0.01)$ and $0.05(p<0.05)$. The mean emotional responses of all emotions and sensory attributes to five flavoured popcorns for each movie were profiled as ratio profiles using Microsoft Office Excel 2021.

\section{Results and discussion}

The results of emotional response to different flavoured popcorns on variety movies were shown in Figures 1 to 5 . Figure 1 shows the mean emotional responses to five flavoured popcorns against 'not eat anything' for the action movie. After consuming butter popcorn, it evoked most of all emotions such as 'daring' 'energetic' 'wild' 'active' and 'pleasant' higher than consuming the other flavoured popcorns significantly ( $p$ $<0.01$ ). While cheese flavoured popcorn, it evoked a few emotions such as 'adventurous' 'whole' and 'steady' higher than butter flavoured popcorn. It seemed these emotions were associated with physical emotion which could get along with the specific characteristic of this movie. Although the mean overall liking scores of all flavoured popcorn were not different, butter and cheese flavoured popcorn fulfilled audiences' feeling for watching action movies better than the others. Especially butter flavoured popcorn, it was an original flavoured popcorn that was served with old-fashioned cowboy and American Indian action movies for a long time (Carlson, 2016). In the cowboy movie, original butter popcorn is often available in Western pubs for a classic cowboy scene (Hofstede, 2016). However, it seemed like butter popcorn consumption made audiences' feeling 'pleasant' when watching the movie but the audiences felt 'guilty' while eating it due to they might be worried about high fat and calories from butter (Levi, 2019) overeating during the entertainment. The researcher would suggest that popcorn producers should avoid communicating butter fortification and advertise that it is only butter flavour margarine and do not include real butter for consumers. Consuming butter flavoured popcorn evoked negative emotional responses as wild and aggressive for action movie watching and may affect the audience's blood running and fighting (Rothkopf and Semlyen, 2021). Toward action movie, the emotional responses evoked by butter and cheese flavour popcorns on 'emotional' attribute more than other flavour popcorns and 'not eat anything' attributes.

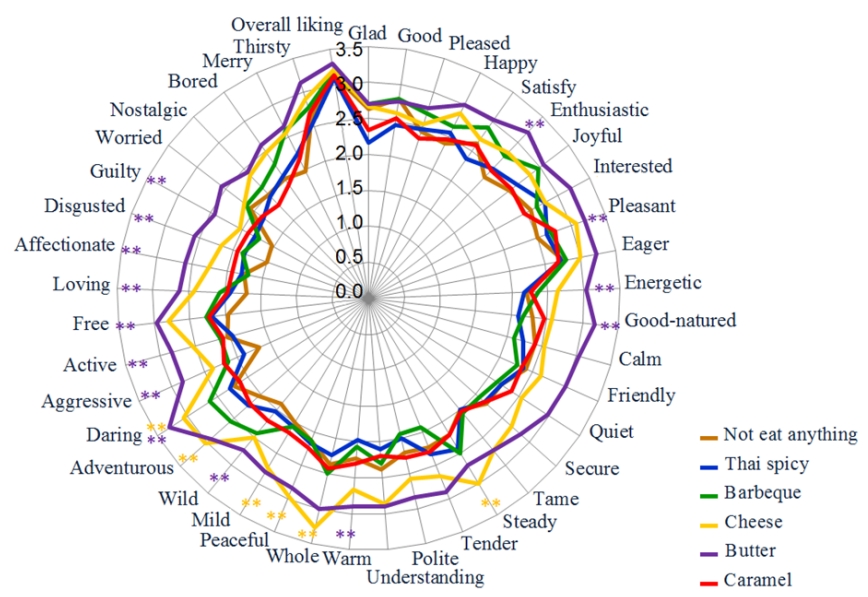

Figure 1. Mean emotion response to different flavoured popcorn compared to 'not eat anything' on watching an action movie.

*Significant at the $0.05, * *$ Significant at the 0.01 .

For comedy movies, after consuming caramel flavoured popcorn, it evoked some emotions such as 'whole' 'warm' 'wild' and 'energetic' higher than 'not eat anything' (Figure 2) and evoked most of the positive emotions higher than other flavoured popcorns. The research done by Noel and Dando (2015) indicated that positive emotions correlate with enhanced sweetness. In addition, the result revealed that caramel flavoured popcorn had the highest mean score on "overall liking" and 'happy' $(p<0.05)$. Cheese flavoured popcorn

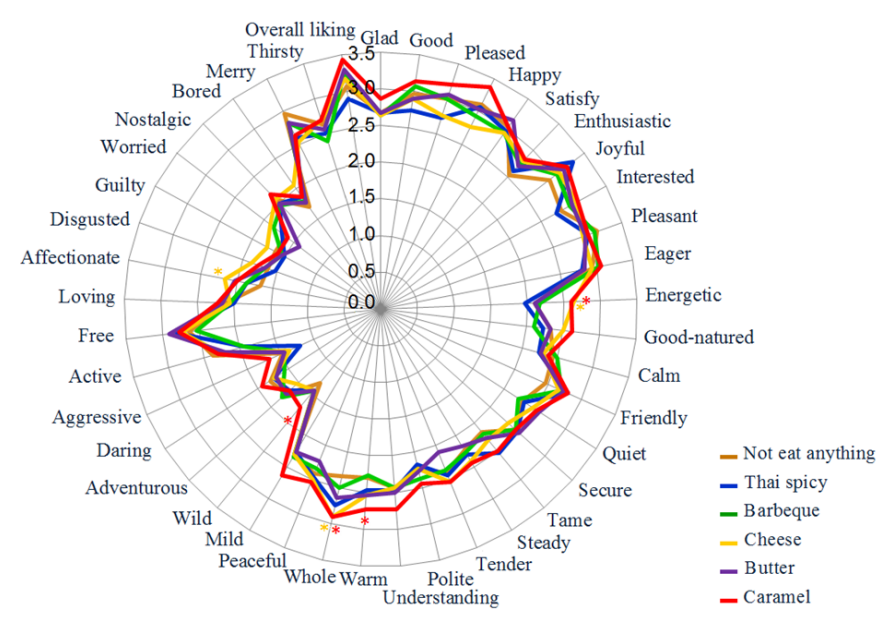

Figure 2. Mean emotion response to different flavoured popcorn compared to 'not eat anything' on watching a comedy movie.

*Significant at the $0.05, * *$ Significant at the 0.01 . 
evoked a few emotions such as 'whole' 'affectionate' and 'energetic'. Thus, caramel and cheese flavoured popcorns could be better choices and were suitable to consume together during comedy movie watching.

In Figure 3, after consuming caramel flavoured popcorn, it evoked some emotional responses such as 'glad' 'calm' 'friendly' 'secure' and 'loving' higher than other flavoured popcorns compared to 'not eat anything' when watching fantasy movie. In addition, the result revealed that caramel flavoured popcorn had the highest mean score on "overall liking" and 'happy' attributes ( $p$ $<0.05$ ). However, 'not to eat anything' evoked the most emotions and was not different from consuming caramel popcorn when watching a fantasy movie. While Tom Yum and barbecue flavoured popcorns evoked most emotions lower than other flavoured popcorns and they had a higher score on the 'thirsty' attribute due to their hot and spicy tastes. Some ingredients like chilli and spices might be an obstacle for fun and excitement toward fantasy movies. It is not surprising to find out that the "not eating anything" attribute has a higher mean score than the 'joyful', 'eager', 'steady', 'adventurous', 'daring', 'free' and 'merry' attributes on watching fantasy movie. It might be true, if the audiences focused on dazzling scenes and exaggerated stories which were outstanding characteristics of the fantasy movie, they are usually not interested to eat anything.

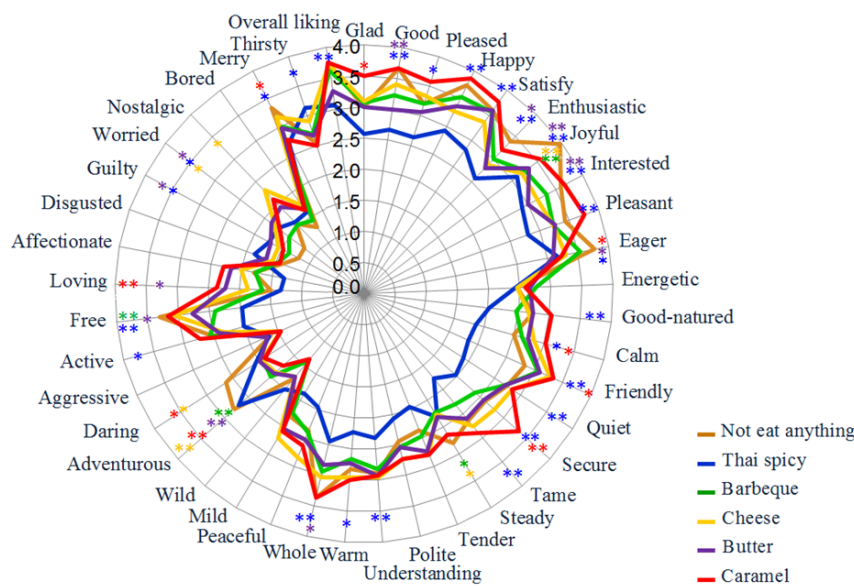

Figure 3. Mean emotion response to different flavoured popcorn compared to 'not eat anything' on watching a fantasy movie.

*Significant at the $0.05, * *$ Significant at the 0.01 .

Watching romance movies and consuming butter popcorn, evoked a few emotions such as 'energetic' and 'pleasant' more than that of other flavoured popcorns obviously $(p<0.01)$ (Figure 4$)$. And the result revealed that butter flavoured popcorn had the highest mean score on 'overall liking' and 'peaceful' attributes. While consuming caramel flavoured popcorn, it evoked a few emotions such as 'pleased' and satisfy' more than the other flavoured popcorn $(p<0.05)$. This result could be described by Mattar et al. (2014) that audiences preferred to eat something sweet immediately on their way out of the romance and horror movies. It is clear that butter and caramel flavour popcorns evoked more emotion than other flavour popcorns and 'not eat anything' for romance movie situations.

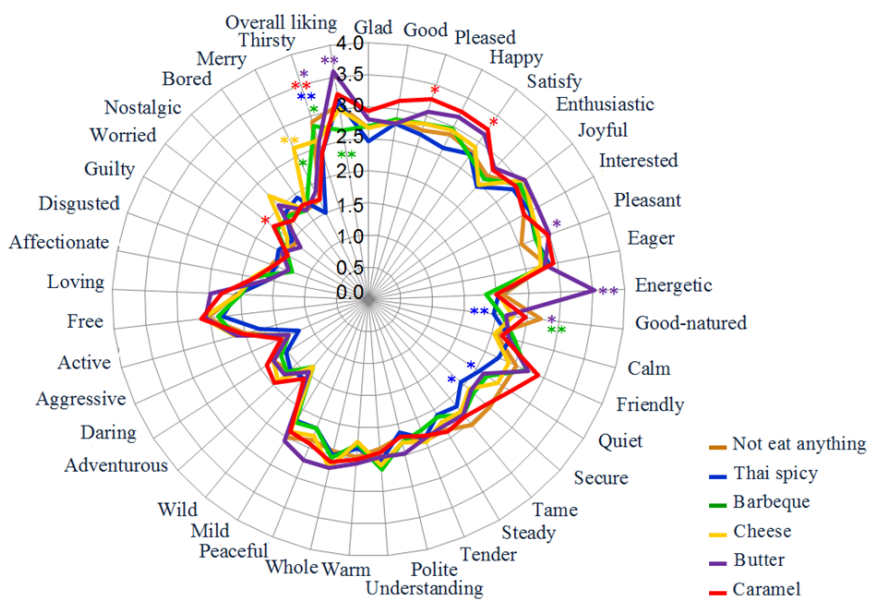

Figure 4. Mean emotion response to different flavoured popcorn compared to 'not eat anything' on watching a romance movie.

*Significant at the $0.05,{ }^{* *}$ Significant at the 0.01 .

Finally, the result after watching a horror movie (Figure 5) showed that caramel flavoured popcorn was more emotional than other flavoured popcorns and 'not eat anything' noticeably $(p<0.01)$. While consuming butter flavoured popcorn, it evoked some emotions such as 'good' 'satisfy' 'joyful' 'friendly' and 'polite' more than 'not eat anything' $(p<0.01)$. However, Zushi et al. (2021) found that the perception of sweetness of food was reduced in the audiences who were watching the horror movie. In conclusion, the audiences who wanted to diet should be careful of eating something sweet during horror movies watching.

While these results could not represent drama, animation and adventure movies but they might infer

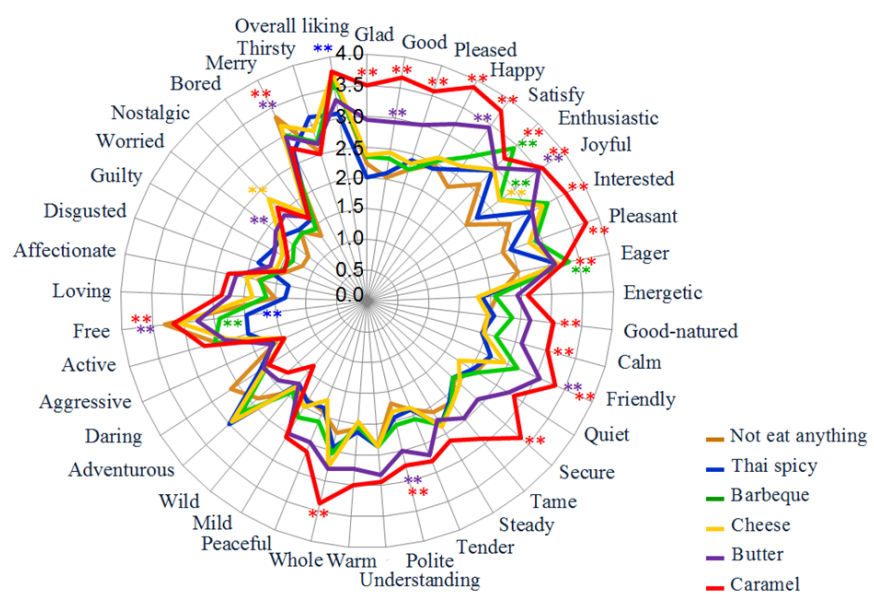

Figure 5. Mean emotion response to different flavoured popcorn compared to 'not eat anything' on watching a horror movie.

*Significant at the $0.05, * *$ Significant at the 0.01 . 
from the similarity to drama-romance, animation-fantasy and adventure-action. However, the emotional responses to these movies would be tested in the next research.

For the reliability of this questionnaire, total-item internal consistency (Cronbach's alpha) was used. The results showed that the total-item Cronbach's alpha of the 41 emotional items when testing with the action, comedy, fantasy, romance and horror movies were more than 0.80 ( $\mathrm{a}=0.95,0.84,0.93,0.80$ and 0.92 , respectively). These results indicated that the internal consistency and reliability of the questionnaires in each of the emotional tests were high [George and Mallery (2003) as stated that the range of between 0.8-0.9 was good reliability].

\section{Conclusion}

Normally, butter and caramel popcorns were the basic flavoured popcorns sold and consumed in the theatres for several movies. Now a day, there were plenty of other flavoured popcorns such as cheese and BBQ presented in the theatres which are accompanied by several soft drinks. In the future, there were a lot of new flavoured popcorns which were developed for consumer choices. Although these new flavours might not give a different taste in the mouth, they might influence emotional responses differently to the consumer. Consumer liking ratings of food products often fail to predict market success. In addition to sensory tests, it was thought that food-evoked emotions provide a sensitive measure to describe products in a way that adds to information from liking. In the case study of the theatre situation, appropriated flavoured popcorns should be served to the individual movies for the positive emotion induction. From this point, some flavoured popcorns with positive psychological benefits should be suitable for some specific movies. Thus, the relationships between taste acceptability and emotional benefits should be used to motive consumer choices in product marketing for the product opportunities and the brand essence. There were a lot of new products in the market that should be promoted taste in the sense of enhancing consumer emotions. In the opinion of the authors, this also had been applied to differentiate consumers for market segmentation using positive emotion or psychology led to better food choices.

\section{References}

Arendt, E. and Zannini, E. (2013). Cereal Grains for the Food and Beverage Industries. $1^{\text {st }} \mathrm{ed}$. Cambridge, United Kingdom: Woodhead Publishing. https:// doi.org/10.1533/9780857098924

Aschemann-Witzel, J., Perez-Cueto, F.J.A.,
Niedzwiedzka, B., Verbeke, W. and Bech-Larsen, T. (2012). Lessons for public health campaigns from analysing commercial food marketing success factors: a case study. BMC Public Health, 12(139), 1 -11. https://doi.org/10.1186/1471-2458-12-139

Bangsuntear, P. (2020). Popcorn. Retrieved on March 24, 2021 from bangkokbiznews Website: https:// www.bangkokbiznews.com/news/detail/862656.

Barthomeuf, L., Rousset, S. and Droit-Volet, S. (2009). Emotion and food. Do the emotions expressed on other people's faces affect the desire to eat liked and disliked food products? Appetite, 52(1), 27-33. https://doi.org/10.1016/j.appet.2008.07.002

Cardello, A.V., Meiselman, H.L., Schutz, H.J., Craig, C., Given, Z., Lesher, L.L. and Eicher, S. (2012). Measuring emotional responses to foods and food names using questionnaires. Food Quality and Preference, 24(2), 243-250. https://doi.org/10.1016/ j.foodqual.2011.12.002

Carlson, J. (2016). A Brief History of Movie Theater Popcorn. Retrieved on September 11, 2019 from Laist Website: https://aist.com/news/food/moviepopcorn-explainer.

Ceylan, M. and Karababa, E. (2004). The effects of ingredients on popcorn popping characteristics. International Journal of Food Science and Technology, 39(4), 361-370. https://doi.org/10.1111/ j.1365-2621.2004.00793.x

Curtis, K.R., McCluskey, J.J. and Wahl, T.I. (2007). Consumer preferences for Western-style convenience foods in China. China Economic Review, 18(1), 1-14. https://doi.org/10.1016/ j.chieco.2006.10.001

George, G. and Mallery, P. (2003). SPSS for Windows step by step: a simple guide and reference 11.0 update. 4th ed., p. 47. Boston, USA: Allyn and Bacon.

Hofstede, D. (2016). 100 Best Westerns Ever Made. Retrieved on February 7, 2021 from Cowboysindians Website: https:// www.cowboysindians.com/2016/11/100-bestwesterns-ever-made.

Jampour, L., Jafarirad, S., Cheraghian, B. and Behrouzian, F. (2019). Effects of Mood Induction Using Movie watching on food intake and hormone levels. Nutrition Today, 54(2), 88-94. https:// doi.org/10.1097/NT.0000000000000329

Khajarern, K., Posri, W. and Duizer, L. (2010). Effects of banana drinks taken for fatigue reduction. International Food Research Journal, 17(1), 181192.

Khajarern, K. (2013). Using sensory and mood 
perceptual maps for generating new energy drink product ideas. International Food Research Journal, 20(1), 175-181.

King, S.C. and Meiselman, H.L. (2010). Development of a method to measure consumer emotions associated with food. Food Quality and Preference, 21(2), 16877. https://doi.org/10.1016/j.foodqual.2009.02.005

Lago, C., Landoni, M., Cassani, E., Doria, E., Nielsen, E. and Pilu, R. (2013). Study and characterization of a novel functional food: purple popcorn. Molecular Breeding, 31(3), 575-85. https://doi.org/10.1007/ s11032-012-9816-6

Levi, A. (2019). Is Butter Actually All That Bad for You? Retrieved on January 10, 2021 from Eatthis Website: https://www.eatthis.com/is-butter-bad-foryou.

Manzano, R., Serra, T. and Gavilán, D. (2019). Sensory Marketing: Straight to the Emotions. Retrieved on May 6, 2019 from IE insight Website: https:// www.ie.edu/insights/articles/sensory-marketingstraight-to-the-emotions.

Mattar, L., Zeeni, N. and Bassil, M. (2014). Effect of movie violence on mood, stress, appetite perception and food preferences in a random population. European Journal of Clinical Nutrition, 69(8), 1-2. https://doi.org/10.1038/ejen.2014.262

Meilgaard, M., Civille, G.V. and Carr, B.T. (2006). Sensory Evaluation Techniques. 4th ed. USA: CRC Press. https://doi.org/10.1201/b16452

Nguyen, V., Cooper, L., Lowndes, J., Melanson, K., Angelopoulos, T.J., Rippe, J.M. and Reimers, K. (2012). Popcorn is more satiating than potato chips in normal-weight adults. Nutrition Journal, 11(71), 1 -6. https://doi.org/10.1186/1475-2891-11-71

Noel, C. and Dando, R. (2015). The effect of emotional state on taste. Appetite, 95, 89-95. https:// doi.org/10.1016/j.appet.2015.06.003

Payne, L.L., Shaw, T. and Caldwell, L.L. (1997). Movies and mood: an exploration of the critical variables related to mood states. Radnor, USA: Northeastern Forest Experiment Station.

Resurreccion, A.V.A. (1998). Consumer Sensory Testing For Product Development. Gaithersburg, USA: Aspen Publication.

Rogers, P.J. (1996). Food choice, mood and mental performance: some examples and some mechanisms. In Meiselman, H.L. and MacFie, H.J.H. (Eds). Food Choice, Acceptance and Consumption, p. 319-345. Weinheim, Germany: Blackie A and P. https:// doi.org/10.1007/978-1-4613-1221-5_9

Rothkopf, J. and Semlyen, P. (2021). The 101 Best Action Movies Ever Made. Retrieved on April 29,
2021 from Timeout Website: https:// www.timeout.com/film/the-101-best-action-moviesever-made.

Singh, J. and Singh, N. (1999). Effects of different ingredients and microwave power on popping characteristics of popcorn. Journal of Food Engineering, 42(3), 161-165. https:// doi.org/10.1016/S0260-8774(99)00114-4

Soylu, S. and Tekkanat, A. (2007). Interactions among expansion volume and kernel properties in various popcorn genogenres. Journal of Food Engineering, 80(1), 336-341. https://doi.org/10.1016/ j.jfoodeng.2006.06.001

Symmank, S., Zahn, S. and Rohm, H. (2018). Visually suboptimal bananas: How ripeness affects consumer expectation and perception. Appetite, 120, 472-81. https://doi.org/10.1016/j.appet.2017.10.002

Wettlaufer, B. and Lemke, S. (2013). PCO group popcorn Nachos equipment. Germany: PCO Group, Dassow.

Wiley, N. (2003). Emotion and film theory. Symbolic Interaction, 26(1), 169-187. https://doi.org/10.1016/ S0163-2396(02)26012-3

Wunsch, N.G. (2020). Sales of the Leading Single-Serve Microwave Popcorn Vendors in the United States in 2020. Retrieved on March 24, 2021 from Statista Website: https://www.statista.com/statistics/ 458440/ us-dollar-sales-of-the-leading-single-servemicrowave-popcorn-vendors.

Zushi, N., Ogawa, M. and Ayabe-Kanamura, S. (2021). Fear reduces perceived sweetness: changes in the perception of taste due to emotional state. SAGE Open, $\quad 11(1), \quad 1-7 . \quad \mathrm{https}: / /$ doi.org/10.1177/2158244021989318 